\title{
PLACENTA PERCRETA. REPORTE DE CASO EN RELACIÓN CON UN PROTOCOLO QUIRÚRGICO OBSTÉTRICO
}

\section{Placenta percreta. A case report with surgical protocoll}

Sergio Enrique Urbina, M.D.*, Pilar Aguilar, M.D.**

Recibido: diciembre 16/2005 - Revisado: febrero 23/2006 - Aceptado: marzo 17/2006

\section{RESUMEN}

La placenta anormalmente adherida es poco común y tiene importancia clínica por su morbimortalidad, a consecuencia de hemorragia, perforación, invasión y lesión de las vías urinarias. Esta adherencia anormal está asociada con la implantación placentaria sobre cicatrices de cesárea previa, incisiones uterinas o legrados. La placenta percreta consiste en la penetración del tejido placentario a través de toda la pared uterina, traspasando la serosa de la misma. La identificación de esta anormalidad antes del parto es posible mediante métodos de imagen (escala de grises por ultrasonido, ecografia Doppler color pulsado o resonancia magnética nuclear).

El tratamiento conservador se acompaña de elevada morbilidad en muchos casos, por lo que el tratamiento quirúrgico se convierte en el definitivo.

La literatura sugiere un aumento previsto en la incidencia de esta condición con base en el incremento del número de cesáreas, por lo que la histerectomía poscesárea será una decisión que enfrentarán los especialistas con mayor frecuencia.

* Coordinador de área de sépticas y cuidado crítico en ginecoobstetricia. Especialista Servicio de Alto Riesgo Obstétrico. Instructor Asistente Facultad de Medicina Fundación Universitaria de Ciencias de la Salud, Departamento de Ginecología y Obstetricia, Hospital de San José. Bogotá, Colombia. Correo electrónico: dr.sergiourbina@supercabletvnet.co

** Residente de Ginecoobstetricia. Fundación Universitaria de Ciencias de la Salud
El caso que se presenta a continuación muestra un escenario en el cual se hace el diagnóstico de placenta percreta y compromiso de la vejiga intraoperatoriamente y describe la preparación preoperatoria de un grupo quirúrgico multidisciplinario y la aplicación de un protocolo, lo cual consideramos fundamental para disminuir la morbimortalidad relacionada con la placenta percreta.

Palabras clave: placenta, percreta, Ultrasonografía de Doppler de Pulso, protocolo quirúrgico, placenta accreta, ultrasonido, Colombia

\section{SUMMARY}

An abnormally attached placenta is unusual, having clinical importance due to mortality arising from consequent haemorrhage, perforation, invasion and injury of the urinary tract. Such abnormal adherence is associated with placental implantation on cicatrices resulting from a previous caesarean operation, uterine incision or curettage. Placenta percreta is defined as penetration of the whole uterine wall's placental tissue, perforating its serous membrane. This abnormality can be identified before birth by imaging methods (ultrasound grey scale, pulsed colour Doppler or nuclear magnetic resonance). Conservative treatment is often inappropriate in these cases and surgical procedure becomes indicated. 
As the literature shows an increase in caesarean sections world-wide, the problem of placenta/accreta is likely to become more common and obstetricians will be faced with the decision to perform hysterectomy after caesarean section.

The case presented here shows how of placenta percreta, with bladder involvement, was intra-operatively diagnosed and describes a multidisciplinary surgical team's preoperative preparation and how a specific protocol was applied. The latter is considered fundamental for avoiding higher morbidity/mortality. Key words: placenta, percreta, Ultrasonography, Doppler, Pulsed, surgical protocol, placenta accreta, ultrasonics, Colombia

\section{CASO CLÍNICO}

Paciente de 26 años, G3, con embarazo de 34,2 semanas por fecha de última regla confiable y ecografías, quien consultó al servicio de urgencias obstétricas por presentar cifras de presión arterial elevadas, asociado a síntomas de vasoespasmo. Como antecedentes de importancia, la paciente había presentado en las dos gestaciones anteriores preeclampsia y se había desembarazado por cesárea en ambas ocasiones.

Se hospitalizó la paciente para realizar más estudios, con paraclínicos iniciales que confirmaron diagnóstico de preeclampsia. Clínicamente no cursó al principio con la forma severa de la enfermedad. Durante la hospitalización se evidenciaron picos altos de presión arterial, exacerbación de síntomas de vasoespasmo y oliguria, a pesar de reanimación volumétrica adecuada. Con base en lo anterior se consideró progresión hacia la forma severa de la enfermedad y se decidió desembarazar. Se solicitó ecografía con escala de grises y Doppler úteroplacentario, los cuales confirmaron placenta corporal anterior y no se demostró signos sugestivos de acretismo.

Con base en los riesgos previstos preoperatoriamente se llevó a cirugía con reserva de concentrado de eritrocitos. Conocidas las limitaciones de la ecografía para descartar completamente el diagnóstico, se preparó el grupo interdisciplinario (obstetricia, cirugía general y urología) ante la eventualidad del hallazgo durante la cirugía, en cuyo caso se seguiría el protocolo de manejo quirúrgico establecido en nuestro servicio para esta entidad, el cual está descrito a continuación.

Se confirmó intraoperatoriamente el diagnóstico de placenta percreta por la apariencia sugestiva de trofoblasto a través de la serosa uterina y la invasión de la vejiga en su aspecto posterior y superior.

De acuerdo al orden de procedimientos se realizó el siguiente protocolo:

1. Anteropulsión del útero grávido, abordaje del retroperitoneo y reparo de arterias ilíacas internas en su origen, en conjunto con el servicio de cirugía general.

2. Cesárea clásica corporal y extracción del recién nacido.

3. Ligadura de arterias hipogástricas, inmediatamente pinzado el cordón umbilical.

4. Histerectomía abdominal subtotal.

5. Cistostomía, exploración de meatos ureterales, confirmando su indemnidad y la permeabilidad ureteral, en conjunto con el servicio de urología. Se dejaron catéteres ureterales “doble J" y sonda de cistostomía.

La evolución transoperatoria fue adecuada, sin

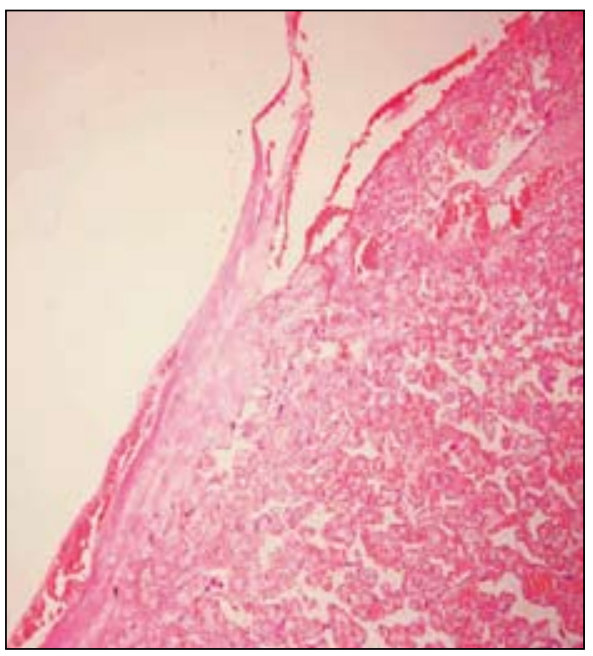

Figura 1. Aspecto microscópico de la pared uterina (10x). Se aprecian fibras musculares lisas miometriales histológicamente normales, infiltradas por numerosas vellosidades coriónicas. 


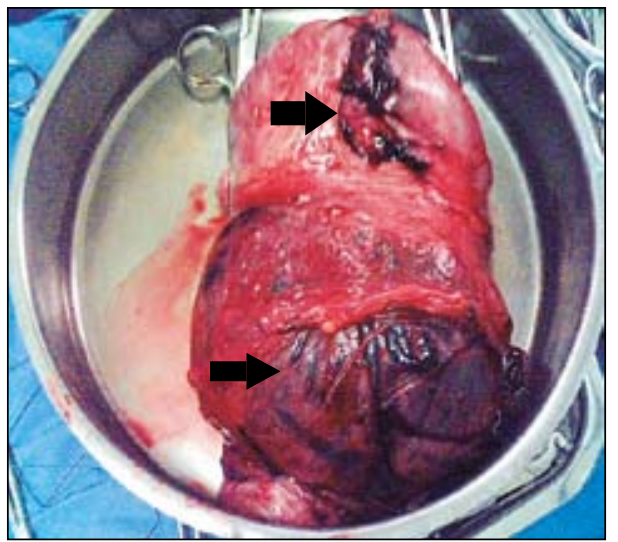

Figura 2. Aspecto macroscópico de la cara anterior del útero. En su parte inferior se aprecia la rotura producida por la placenta percreta, la cual emerge a través de la serosa uterina (flecha inferior). A nivel fundocorporal se aprecia la histerotomía realizada para la extracción del neonato (flecha superior).

inestabilidad hemodinámica, ni sospecha clínica de coagulopatía. Los requerimientos de sangre perioperatoriamente se limitaron a 2 unidades de concentrado de eritrocitos.

La evolución de la paciente en el posoperatorio fue satisfactoria, con funciones renal, hematológica y de la coagulación adecuadas, sin deterioro clínico ni paraclínico durante su estancia hospitalaria.

Se dio el alta hospitalaria al cuarto día posoperatorio, con sonda vesical durante 14 días. No requirió nueva hospitalización. El estudio histopatológico confirmó el diagnóstico de placenta percreta.

\section{DISCUSIÓN}

La placenta accreta tiene una frecuencia que varía entre 10 y 48 por 10.000 partos. ${ }^{1,2}$

El acretismo se clasifica de acuerdo a un espectro que involucra tres posibles condiciones. La placenta accreta está limitada al miometrio superficial, la placenta increta implica el compromiso de todo el espesor miometrial y la placenta percreta corresponde al mayor trastorno adherencial placentario sobre el útero. La adherencia se produce de tal manera, que la placenta penetra y excede el continente uterino, involucrando los órganos y tejidos vecinos, en especial la vejiga. Parece un cuadro infrecuente, pero su incidencia es probablemente mayor puesto que no todos los casos son publicados y, aunque el riesgo de daño del ureter y vejiga es tan solo del 2$3 \%$, se hace necesaria la intervención de personal especializado para el correcto manejo quirúrgico, como se documentó en nuestro caso.

El diagnóstico con la ecografía y el Doppler es relativamente fácil, pero requiere de la experiencia de personal entrenado. La sensibilidad reportada en los estudios es variable, desde el 2,5\% en una revisión de 310 casos durante 10 años $^{3}$ hasta el 82,4\% en un estudio clínico de pacientes con alto riesgo de acretismo. ${ }^{4}$ La resonancia magnética nuclear también se ha utilizado para el diagnóstico de acretismo placentario y los reportes muestran diferente sensibilidad y especificidad del método. ${ }^{5}$ Dada esta variabilidad y la necesidad de observadores muy entrenados para hacer el diagnóstico, sigue siendo frecuentemente un hallazgo intraoperatorio; en cuyo caso la experiencia del cirujano y demás personal debe ser óptimo para disminuir la morbimortalidad.

El manejo quirúrgico debe estar protocolizado en conjunto con los servicios de cirugía general y urología. Teniendo en cuenta los inconvenientes para el abordaje quirúrgico y el exuberante flujo arterial placentario, implica una pérdida hemática considerable, requiriendo transfusión en la mayoría de los casos. ${ }^{6}$

Con base en lo anterior y de acuerdo con otros reportes, consideramos que la presencia de un grupo interdisciplinario, el seguimiento del protocolo quirúrgico propuesto y la reserva preoperatoria de derivados sanguíneos son elementos determinantes para disminuir la morbimortalidad relacionada con la placenta percreta. ${ }^{1,7}$

Se han documentado diferentes modalidades de manejo conservador, dejando la placenta in situ y usando metotrexate, mediante embolización de las arterias hipogástricas, las uterinas y las implicadas en el sangrado intraoperatorio, las cuales son utilizadas para conservar el útero y la fertilidad. Estos estudios han reportado una variada frecuencia de 
resultados adversos. ${ }^{8-10}$ Por lo tanto, consideramos que el tratamiento definitivo es el quirúrgico con histerectomía, obteniendo resultados satisfactorios y evitando eventos catastróficos posteriores como la miometritis y el choque séptico.

No existen evidencias de nivel I o II para recomendar algún tipo de histerectomía en particular. Diferentes autores recomiendan la histerectomía subtotal considerando que es menos dispendiosa, lo que disminuye el tiempo quirúrgico y la pérdida sanguínea y que puede relacionarse con un menor número de lesiones de las vías urinarias. ${ }^{11}$

De acuerdo a la evolución del caso, el tratamiento de la paciente fue oportuno y adecuado, basado principalmente en el protocolo anotado. Debido a estas medidas, no se documentó morbilidad grave ni mortalidad materna o perinatal.

\section{AGRADECIMIENTOS}

Dra. Pilar Archila

Jefe Departamento de Patología. Hospital de San José. Bogotá.

Dra. Patricia Cruz.

Residente de Patología. Hospital de San José. Bogotá.

\section{REFERENCIAS}

1. Hung TH, Shau WY, Hsieh CC, Chiu TH, Hsu JJ, Hsieh TT. Risk factors for placenta accreta. Obstet Gynecol 1999;93:545-50.

2. Sánchez LM, Angel E, Castro E. Placenta acreta en el primer trimestre de la gestación: presentación de dos casos y revisión de la literatura. Rev Colomb Obstet Ginecol 2000;51:258-63
3. Gielchinsky Y, Rojansky N, Fasouliotis SJ, Ezra Y. Placenta accreta-- summary of 10 years: a survey of 310 cases. Placenta 2002;23:210-4.

4. Chou MM, Ho ES, Lee YH. Prenatal diagnosis of placenta previa accreta by transabdominal color Doppler ultrasound. Ultrasound Obstet Gynecol 2000;15:28-35.

5. Lam G, Kuller J, Mc Mahon M. Use of magnetic resonance imaging and ultrasound in the antenatal diagnosis of placenta accreta. J Soc Gynecol Investig 2002;9:37-40.

6. Weiniger CF, Elram T, Ginosar Y, Mankuta D, Weissman C, Ezra Y. Anaesthetic management of placenta accreta: use of a pre-operative high and low suspicion classification. Anaesthesia 2005;60:1079-84.

7. Coates VA, Fishman MS, McCall WG. Placenta percreta: report of a case. CRNA 1999;10:165-9.

8. Levine AB, Kuhlman K, Bonn J. Placenta accreta: comparison of cases managed with and without pelvic artery balloon catheters. J Matern Fetal Med 1999;8:173-6

9. Courbiere B, Bretelle F, Porcu G, Gamerre M, Blanc B. J Gynecol Obstet Biol Reprod (Paris) 2003;32:549-54.

10. Davis JD, Cruz A. Persistent placenta increta: a complication of conservative management of presumed placenta accreta. Obstet Gynecol 1996t;88:653-4.

11. Thakar R, Ayers S, Clarkson P, Stanton S, Manyonda I. Outcomes after total versus subtotal abdominal hysterectomy. N Engl J Med 2002; 347:1318-25.

12. Beukenholdt RW, Allman A. Undiagnosed placenta percreta. J Obstet Gynaecol 2002;22:688.

13. Takai N, Eto M, Sato F, Mimata H, Miyakawa I. Placenta percreta invading the urinary bladder. Arch Gynecol Obstet 2005;271:274-5.

14. ACOG Committee opinion. Number 266, January 2002: placenta accreta. Obstet Gynecol 2002;99:169-70. 